\title{
Juxta-membrane S-acylation of plant receptor-like kinases - fortuitous or functional?
}

Charlotte H. Hurst ${ }^{1,2}$, Kathryn M. Wright ${ }^{2}$, Dionne Turnbull ${ }^{1}$, Kerry Leslie ${ }^{1,2,3}$, Susan Jones ${ }^{2}$, and Piers A. Hemsley ${ }^{1,2, *}$

1. Division of Plant Science, School of Life Science, University of Dundee (at JHI), Invergowrie, Dundee DD2 5DA, UK.

2. Cell and Molecular Science, James Hutton Institute, Invergowrie, Dundee DD2 5DA, UK

3. Present address: Biomedical Sciences Research Complex, University of St Andrews, St Andrews, KY16 9ST, UK.

* Author for correspondence: p.a.hemsley@dundee.ac.uk

\section{Abstract}

S-acylation is a common post-translational modification of membrane protein cysteine residues with many regulatory roles. S-acylation adjacent to transmembrane domains has been described in the literature as affecting diverse protein properties including turnover, trafficking and microdomain partitioning. However, all of these data are derived from mammalian and yeast systems. Here we examine the role of S-acylation adjacent to the transmembrane domain of the plant pathogen perceiving receptor-like kinase FLS2. Surprisingly, S-acylation of FLS2 adjacent to the transmembrane domain is not required for either FLS2 trafficking or signalling function. Expanding this analysis to the wider plant receptor-like kinase superfamily we find that S-acylation adjacent to receptor-like kinase domains is common but poorly conserved between orthologues through evolution. This suggests that $\mathrm{S}$-acylation of receptor-like kinases at this site is likely the result of chance mutation leading to cysteine occurrence. As transmembrane domains followed by cysteine residues are common motifs for Sacylation to occur, and many S-acyl transferases appear to have lax substrate specificity, we propose that many receptor-like kinases are fortuitously S-acylated once chance mutation has introduced a cysteine at this site. Interestingly some receptor-like kinases show conservation of S-acylation sites between orthologues suggesting that S-acylation has come to play a role and has been positively selected for during evolution. The most notable example of this is in the ERECTA-like family where Sacylation of ERECTA adjacent to the transmembrane domain occurs in all ERECTA orthologues but not in the parental ERECTA-like clade. This suggests that ERECTA S-acylation occurred when ERECTA emerged during the evolution of angiosperms and may have contributed to the neo-functionalisation of ERECTA from ERECTA-like proteins.

\section{Introduction}

S-acylation is a post-translational protein modification where fatty acids, such as palmitate or stearate, are covalently linked to specific cysteines through thioester linkages. No consensus sequence for $\mathrm{S}$ acylation has been described and the outputs of prediction software such as CSS-Palm [1] are still some way off from being acceptably reliable. However, one structural motif has emerged; a cysteine at the cytoplasmic face of a transmembrane domain. In mammalian systems, S-acylation at cysteine residues adjacent to the transmembrane domains of single pass receptor-like portions appears to be a common feature and is important for protein functionality. Juxta-transmembrane S-acylation has been shown to affect Toll-like receptor (TLR2) trafficking to the plasma membrane and therefore regulate T-cell signalling [2]. S-acylation of the anthrax toxin receptor, TEM8, spatially segregates it in the plasma membrane away from its cognate E3 ubiquitin ligase, $\mathrm{Cbl}$, to control TEM8 endocytosis 
and targeting to the lysosomes [3]. LRP6 is involved in the Wnt signalling pathway and requires Sacylation of the juxta-transmembrane (juxta-TM) cysteines [4]. S-acylation induces tilting of the LRP6 transmembrane span to prevent exposure of hydrophobic amino acids to the aqueous environment during trafficking through the endoplasmic reticulum. S-acylation-deficient LRP6 is recognised by the endoplasmic reticulum quality control mechanisms due to hydrophobic mismatch and targeted for ubiquitin-mediated degradation [4]. We recently identified numerous proteins from Arabidopsis as being S-acylated through a proteomics screen [5]. Included in this group of S-acylated proteins were members of the receptor-like kinase (RLK) superfamily. Receptor-like kinases are related to mammalian single pass receptor-like proteins such as TLR2 [6], and S-acylation of receptor-like kinases has been assumed to function in a similar manner to that described for single pass transmembrane proteins in mammals. However, given the evolutionary distance between plants and animals, and the differences in membrane compositions, it should not be presumed that juxta-transmembrane domain S-acylation has the same effect in both plant and animal kingdoms.

RLKs are one of the largest gene families in Arabidopsis with over 610 members [6] and their roles in a wide variety of plant processes have been well-characterised. This includes plant growth and development through the brassinosteroid hormone receptor BRI1 and its co-receptor BAK1 [7], and the control of organ formation and cell fate specification through ERECTA [8]. Other RLKs such as FERONIA and ERULUS are required for brassinosteroid-mediated cell expansion and pollen and root hair elongation $[9,10]$. Additionally, wall-associated kinases (WAKs) interact with the cell wall and are required for cell expansion during plant development [11]. RLKs are also involved in abiotic stress responses through RPK1 which regulates $A B A$ and osmotic stress signalling, and GHR1 which controls ABA- and $\mathrm{H}_{2} \mathrm{O}_{2}$-regulated anion channels in guard cells. Other members of the RLK superfamily are involved in plant-microbe interactions: Xa21 from Oryza sativa was the first RLK characterised in plants and is involved in bacterial pathogen resistance [12]. Other well-characterised receptors, FLS2 [13] and EFR [14] perceive the bacterial-derived peptides flagellin and elongation factor-Tu, respectively, while CERK1 and LYK4 are involved in recognition of chitinous fungal elicitors [15]. While much is known about members of the RLK superfamily, little is known about the specific role of S-acylation in RLK function.

Based on data from the original proteomics screen, we identified juxta-TM S-acylation sites in the model RLK FLS2 [5]. These data have subsequently been validated using orthogonal metabolic labelling strategies putting S-acylation of FLS2 at these sites beyond reasonable doubt [16]. Both studies demonstrated that mutation of these cysteines abolished S-acylation and we described a mild effect of their mutation on AtFLS2- mediated processes [5]. However, this work was performed with mGFP6 tagged versions of AtFLS2 expressed from the $35 \mathrm{~S}$ promoter. We have since determined that many tags, when appended to AtFLS2, impact unpredictably on AtFLS2 function, with mGFP6 in the context of the pMDC vector series [17] used being particularly deleterious to AtFLS2 function [18]. Some of these effects can apparently be masked by dosage compensation [18] such as the 355 promoter used in our original study [5]. We therefore decided to revisit our earlier work in greater depth using untagged forms of AtFLS2 expressed close to native levels to accurately gauge the role and contribution of juxta-TM S-acylation to AtFLS2 function. 
bioRxiv preprint doi: https://doi.org/10.1101/693887; this version posted July 5, 2019. The copyright holder for this preprint (which was not certified by peer review) is the author/funder, who has granted bioRxiv a license to display the preprint in perpetuity. It is made available under aCC-BY-NC 4.0 International license.

\section{Results}

\section{Juxta-transmembrane domain S-acylation is not required for AtFLS2 function}

We previously proposed that loss of AtFLS2 juxta-transmembrane (juxta-TM) S-acylation in AtFLS2 $\mathrm{C}^{830,831} \mathrm{~S}$ mutants had a mild negative effect on flagellin signalling outputs when expressed as Cterminal mGFP6 fusions at 100-300x normal levels [5]. We have since determined that epitope fusions, in particular mGFP6, can have deleterious and unpredictable effects on AtFLS2 function when AtFLS2 is expressed at physiological levels, calling the biological relevance of these data into question [18]. To determine whether the minor difference in AtFLS2 function observed between AtFLS2 and AtFLS2 $C^{830,831} \mathrm{~S}$ mGFP6 tagged lines was genuine we generated fls2/proAtFLS2:AtFLS2 [18] and fls2/proAtFLS2:AtFLS2 $C^{830,831} \mathrm{~S}$ lines and selected plants showing similar AtFLS2 and AtFLS2 $\mathrm{C}^{830,831} \mathrm{~S}$

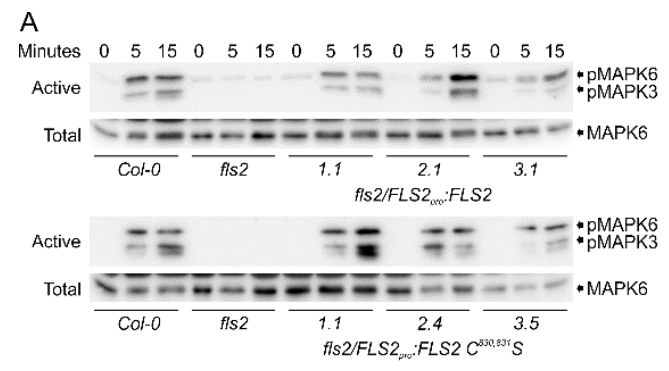

B

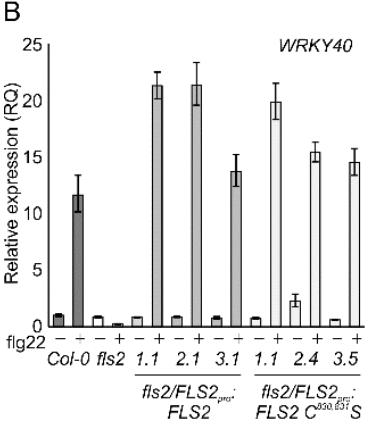

$\mathrm{C}$

E
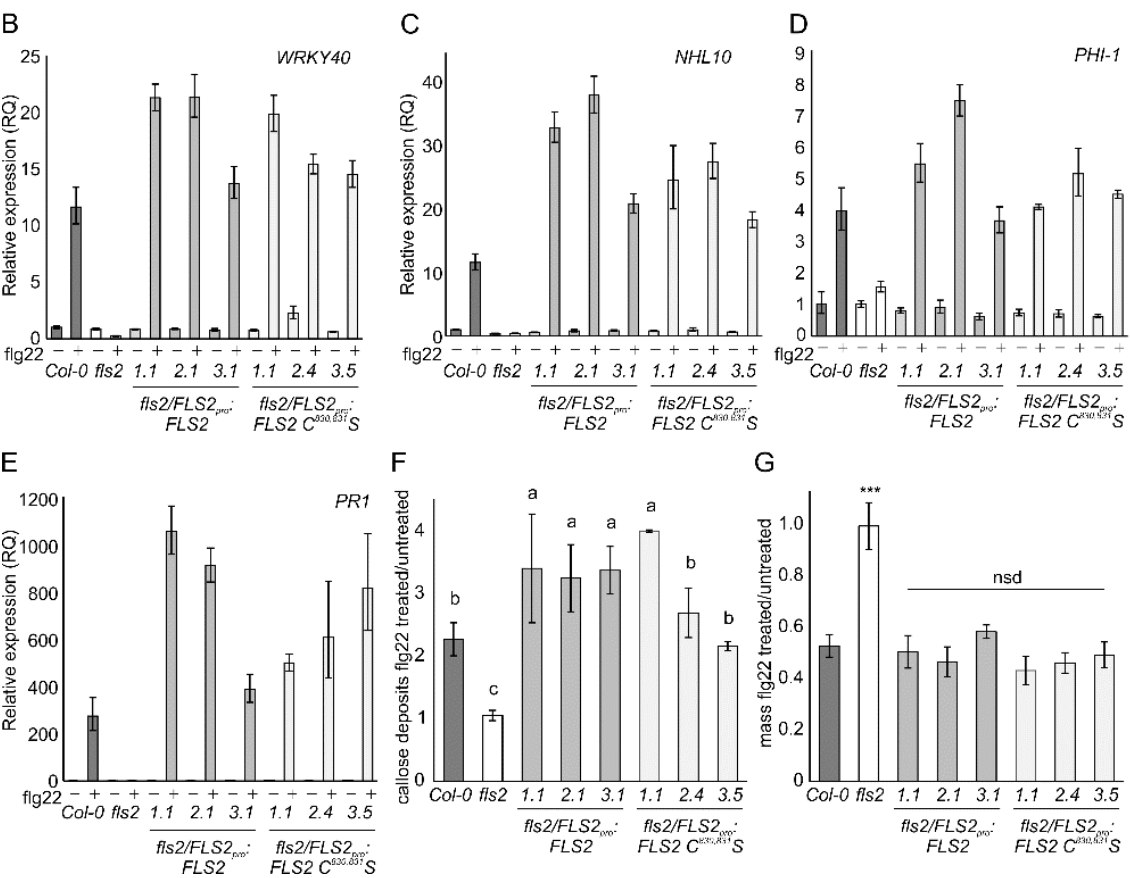

G

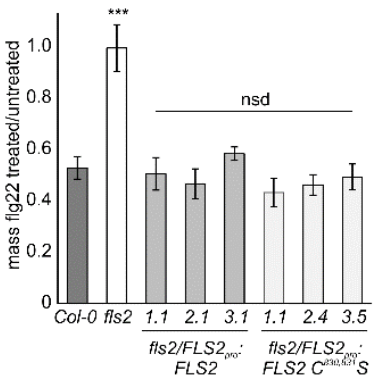

Figure 1. FLS2 $\mathrm{C}^{830,831} \mathrm{~S}$ expressing plant responses to flg22 stimulation are indistinguishable from wild type (A). MAPK activation in fls2/FLS2pro:FLS2 and fls2/FLS2pro:FLS2 $C^{830,831} S$ seedlings in response to $100 \mathrm{nM}$ flg22 over time as determined by immunoblot analysis. pMAPK6/pMAPK3 show levels of active form of each MAPK. MAPK6 indicates total levels of MAPK6 as a loading control. Upper shadow band in MAPK6 blot is rubisco detected non-specifically by secondary antibody. (B). Induction of NHL10, (C) WRKY40 and (D) PHI-1 gene expression after 1 hour treatment with $1 \mathrm{mM}$ flg22 in fls2/FLS2pro:FLS2 and fls2/FLS2pro:FLS2 $C^{830,831}$ s seedlings as determined by qRTPCR. (E) Induction of PR1 gene expression after 24 hours treatment with 1mM flg22 in fls2/FLS2pro:FLS2 and fls2/FLS2pro:FLS2 C830,831S seedlings as determined by qRT-PCR. Values were calculated using the DDCT method from 3 technical replicates, error bars represent $R Q_{\text {MIN }}$ and $R Q_{\text {MAx }}$ and constitute the acceptable error level for a $95 \%$ confidence interval according to Student's t-test. Similar data were obtained over 2 biological repeats (additional data shown in supplemental figure 1). (F) Relative quantification of callose deposition following 12 hours treatment with water or $1 \mathrm{mM}$ flg22. Averages are shown from at least 2 biological repeats, error bars represent SEM and statistically similar groupings are denoted by a, b, c as determined by ANOVA and post-hoc Tukey-Kramer test. (G) Average relative seedling mass (flg22 treated/untreated) of seedlings grown in $1 \mu \mathrm{m}$ flg22 for $10 \mathrm{~d}$ (14 d post germination). Data are averages of three independent biological replicates. Error bars show se. Asterisks denote statistically significant differences compared with that of the Col0 control $(* * *, \mathrm{P}<0.01)$ determined by one-way ANOVA and Tukey's HSD test. 
protein expression levels (Supplemental figure 1A). Following flg22 perception, AtFLS2 heterodimerises with its co-receptor BAK1 leading to the formation of an active signalling complex. This signalling complex initiates complex intracellular signalling cascades to promote plant immunity. MAPK activation is one of the earliest detectable signalling events following flg22 perception [19]. After flg22 treatment, fls2c/AtFLS2 pro:AtFLS2 and fls2c/AtFLS2:AtFLS2 $C^{830,831} S$ plant line MAPK6/3 activation was indistinguishable from Col-0, while fls2c lines showed no MAPK6/3 activation (Fig $1 \mathrm{~A}$ ). MAPK-dependent genes (NHL10, WRKY40) were similarly activated in Col-0, fls2c/AtFLS2 pro:AtFLS2 and fls2C/AtFLS2:AtFLS2 $C^{830,831} \mathrm{~S}$ plant lines (Fig 1B, C, Supplemental fig 1B, C). To test whether other signalling pathways were affected by loss of AtFLS2 juxta-TM S-acylation, $\mathrm{PHI}-1$, a $\mathrm{Ca}^{2+}$ mediated flg22inducible defence marker gene, was examined. Again, $P H I-1$ induction was elevated in fls2c/AtFLS2:AtFLS2 $C^{830,831} S$ plants to a similar degree as Col-0 and fls2c/AtFLS2 pro:AtFLS2 control lines (Fig 1D, Supplemental fig 1D). PR1, a marker of salicylic acid (SA) mediated gene induction and sustained signalling, was unaffected in fls2c/AtFLS2:AtFLS2 $C^{830,831} S$ plant lines (Fig 1E, Supplemental figure $1 \mathrm{E}$ ). Following longer term exposure to flg22 SA signalling is elevated and callose, a $\beta$-glucan polysaccharide, is deposited to reinforce the cell wall and prevent bacterial penetration. Callose

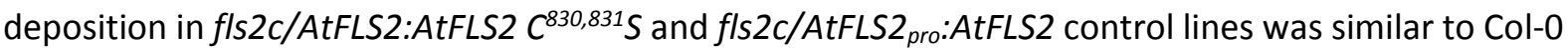
and in all cases much greater than in fls $2 c$ (Fig $1 \mathrm{~F}$, Supplemental fig $1 \mathrm{~F}$ ). This suggests that loss of the juxta-TM cysteines does not adversely impact any flg22-mediated AtFLS2 signalling outputs. One of the responses to prolonged flg22 exposure is the inhibition of seedling growth, and this is often used as a proxy for the overall combined signalling outputs and long-term effects of AtFLS2 activation. Growth inhibition experiments showed no deleterious effects of AtFLS2 $\mathrm{C}^{830,831} \mathrm{~S}$ on seedlings growth inhibition responses compared to Col-0, or AtFLS2 expressing control lines (Fig 1G). This suggests that,

A
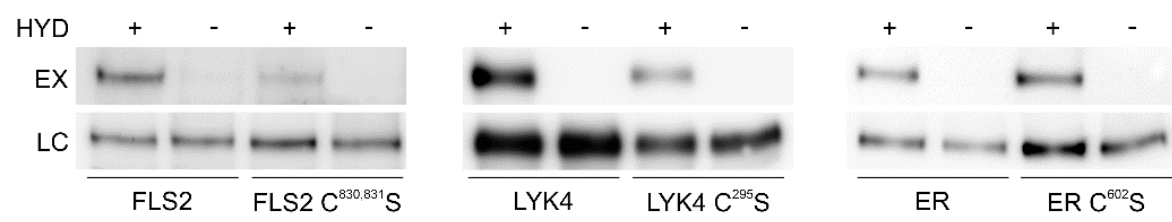

B
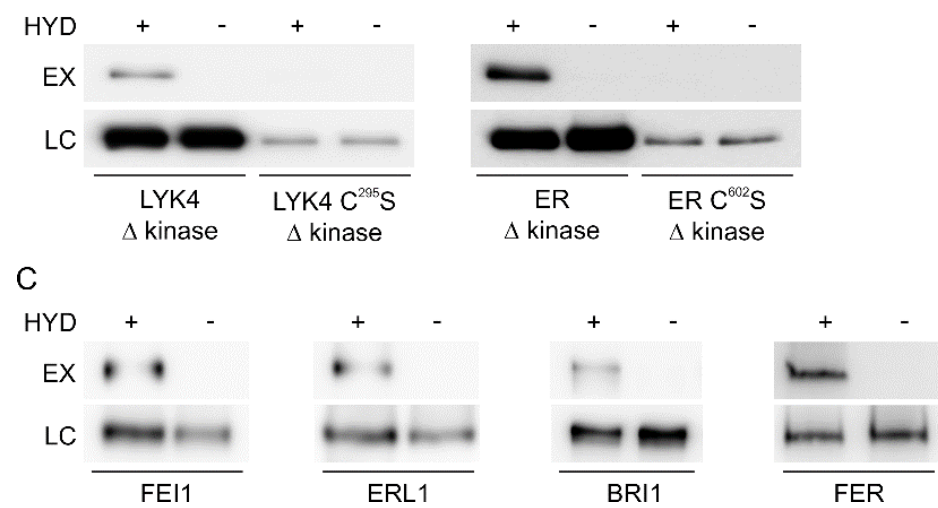

Figure 2. Receptor-like kinases are S-acylated at sites other than the transmembrane region. (A) Receptor-like kinases show reduced but readily apparent S-acylation following mutation of juxta-TM cysteine residues. S-acylation state of receptor-like kinases was determined using Acyl-biotin exchange (ABE; FLS2 variants expressed in Arabidopsis) or Acyl-RAC (LYK4 and ER variants expressed in $N$. benthamiana) methods. (B) Truncations of LYK4 and ER indicate that S-acylation occurs at juxta-TM cysteines similarly to FLS2. (C) Receptor-like inases that do not contain juxta-TM cysteines are also S-acylated. HYD indicates presence (+) or absence (-) of hydroxylamine required for S-acyl group cleavage during the thiopropyl Sepharose $6 \mathrm{~b}$ capture (Acyl-RAC) or biotinylation step (ABE) step. Thiopropyl Sepharose 6b (Acyl-RAC) or neutravidin (ABE) enriched proteins were eluted and represent S-palmitoylated proteins (EX). Prior to thiopropyl Sepharose 6b (Acyl-RAC) or neutravidin (ABE) capture a sample was removed as an input loading control (LC). 
contrary to previous conclusions from using tagged AtFLS2 $\mathrm{C}^{830,831} \mathrm{~S}$ lines [5], juxta-transmembrane Sacylation of AtFLS2 is not required for function.

\section{RLK superfamily S-acylation is not confined to juxta-TM cysteines}

The above findings on AtFLS2 juxta-TM S-acylation led us to question why AtFLS2 is S-acylated at these sites if, unlike reported juxta-TM S-acylation in animals $[3,4,20]$, it has no discernible effect on function. In a proteomic screen for S-acylated proteins [5] we identified 16 receptor-like kinases from Arabidopsis (Supplemental Table 1) that, like AtFLS2, all contain at least one cysteine within 5 amino acids of the C-terminus of their single transmembrane domain (based on definitions from the Aramemnon database [21]). This strongly suggests that, like AtFLS2, these cysteines are likely sites of $\mathrm{S}$-acylation. Analysis of the juxta-TM region of all RLK superfamily members in the Arabidopsis genome [6] (Supplemental figure S2) suggest that in total 108 contain cysteines adjacent to the TM (at or within the 5 cytoplasmic amino acids following the TM/cytoplasm interface). These cysteines represent high probability candidate sites for S-acylation [3]. The RLKs containing these cysteines are distributed widely throughout the Arabidopsis RLK superfamily (Supplemental Table 2). Two well studied receptor-like kinases that contain juxta-TM cysteines, AtLYK4 and AtERECTA, were cloned and, like AtFLS2, were found to be positive for S-acylation (Fig 2A). Using a recently devised and more sensitive S-acylation assay [22] to determine the S-acylation state of AtFLS2 expressed at native levels in Arabidopsis we observed that although S-acylation was markedly reduced in the AtFLS2 $C^{830,831} \mathrm{~S}$ mutant it was not abolished as previously described [5, 16]. This suggests that AtFLS2 contains additional or alternative S-acylation sites with low occupancy. We therefore expressed AtLYK4 and AtERECTA as EGFP fusions alongside versions containing juxta-TM cysteine mutations (AtLYK4 $\mathrm{C}^{295} \mathrm{~S}$, AtERECTA $\mathrm{C}^{602} \mathrm{~S}$ ) in $\mathrm{N}$. benthamiana and assessed S-acylation state. In both cases S-acylation was still observed in the cysteine to serine mutants (Fig 2A). To verify that the juxta-TM cysteine in AtERECTA and AtLYK4 is truly a site of S-acylation, minimal constructs corresponding to AtERECTA, AtERECTA $\mathrm{C}^{602} \mathrm{~S}$, AtLYK4 and AtLYK4 $\mathrm{C}^{295} \mathrm{~S}$ but lacking the kinase domain were generated. In these constructs the juxta-TM cysteines are the only cysteines remaining in the cytoplasmic portion of the protein and are therefore the only cysteines that could become S-acylated. Testing of these constructs for S-acylation confirms that the juxta-TM cysteines in AtLYK4 and AtERECTA are S-acylated (Fig 2B). All of these data combined strongly support the hypothesis that AtFLS2, AtLYK4 and AtERECTA contain S-acylation site(s) in addition to the S-acylation site adjacent to the transmembrane domain. These sites may therefore also exist within receptor-like kinases that do not contain juxta-TM cysteines. To test this we cloned FEI1, FER (Feronia), AtERL1 (AtERECTA-like 1) and BRI1 (Brassinosteroid insensitive 1) as examples of receptor-like kinases that do not contain juxta-TM cysteines and assessed them for $\mathrm{S}$ acylation. Again we found that S-acylation was a feature of all RLKs tested (Fig $2 \mathrm{C}$ ) suggesting that Sacylation of the RLK superfamily is much more common than previously proposed [5], possibly even ubiquitous, and occurs at sites other than juxta-TM cysteines.

\section{Juxta-transmembrane domain cysteines does not control receptor-like kinase localisation}

Data from mammalian and yeast systems suggests that juxta-TM cysteine S-acylation can help direct the export of correctly folded proteins from the Golgi or ER to the plasma membrane, and prevent hydrophobic mismatch between the TM helix and the Golgi/ER membranes leading to protein turnover [4]. Our previous data suggested that AtFLS2 S-acylation was not required for PM targeting [5] and the data presented above indicate that AtFLS2 with mutated juxta-membrane S-acylation sites is fully functional and must therefore be at the plasma membrane in Arabidopsis. To determine 
bioRxiv preprint dol: https://doi.org/10.1101/693887; this version posted July 5, 2019. The copyright holder for this preprint (which was not certified by peer review) is the author/funder, who has granted bioRxiv a license to display the preprint in perpetuity. It is made available under aCC-BY-NC 4.0 International license.

whether the lack of effect of juxta-TM S-acylation on localisation was restricted to AtFLS2 we tested whether AtERECTA and AtLYK4 required S-acylation to traffic to the PM. Confocal imaging indicates

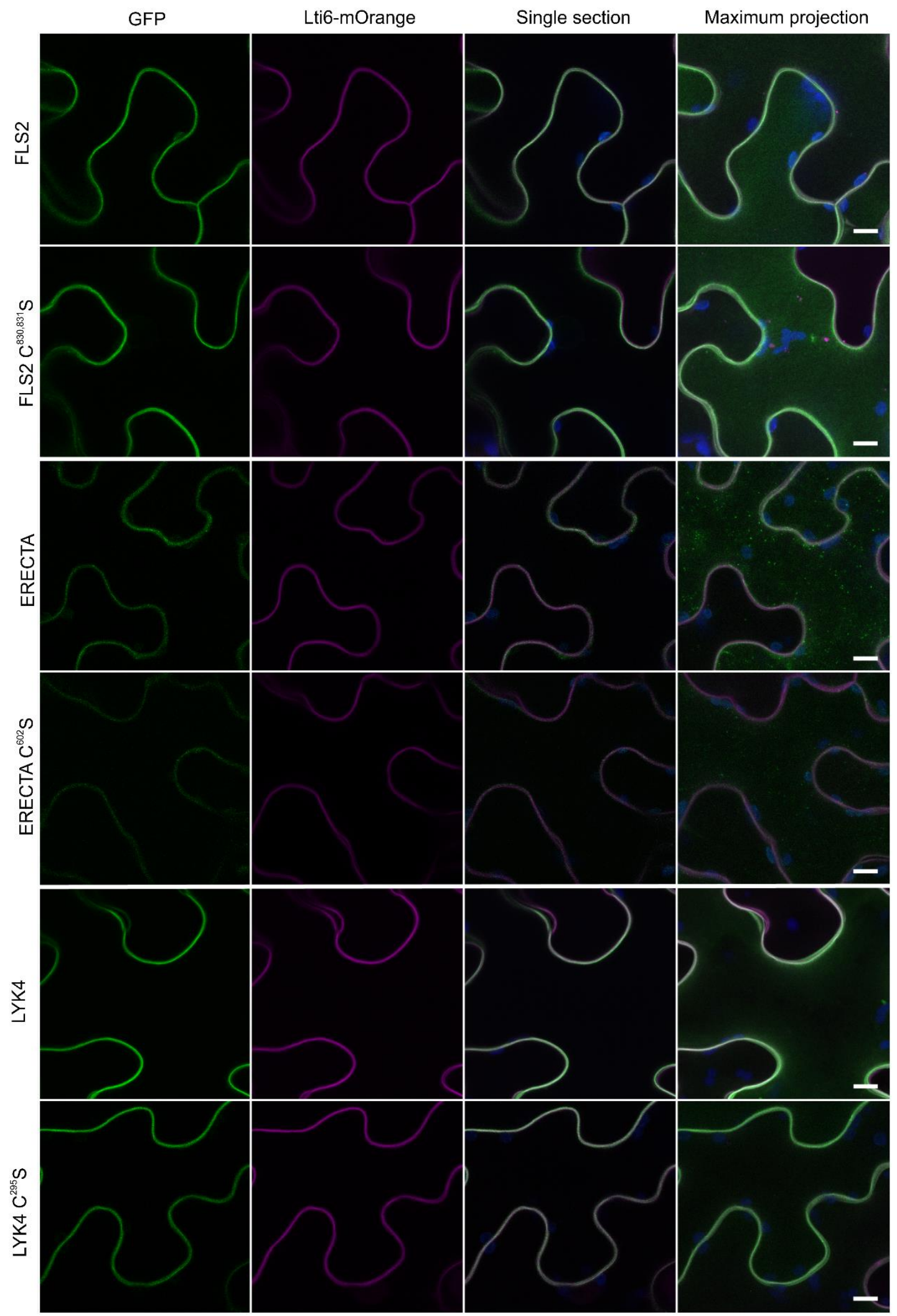

Figure 3. Receptor-like kinase localisation to the plasma membrane does not require S-acylation. Receptor-like kinase variants fused to EGFP (green) were imaged in mOrange-Lti6b (magenta) expressing plants. Chlorophyll auto-fluorescence is shown in blue. Scale bar represents $10 \mu \mathrm{m}$. 
that AtFLS2, AtLYK4, and AtER traffic to the PM in the absence of juxta-TM cysteines, with the intracellular distribution of the juxta-TM mutant forms being indistinguishable from wild type (figure 3).

\section{Juxta-membrane S-acylation in not necessarily conserved between orthologues}

If juxta-TM S-acylation were major or essential determinants of a receptor-like kinases' function it would be expected that juxta-TM sites of S-acylation would be under strong selective pressure over evolutionary time. As a result, clear patterns of gain or loss of S-acylation would be expected with the presence/absence of juxta-TM S-acylation being highly conserved between RLK orthologues. If juxtaTM cysteines occur through random mutation and confer no particular benefit, then they will be under neutral selection and their presence would be more or less random with no clear evolutionary pattern. Using the Aramemnon database of angiosperm membrane proteins we examined orthologues of Arabidopsis RLKs containing juxta-TM cysteines found both through proteomics or tested here for Sacylation, and examined them for juxta-TM cysteines. As can be seen in supplemental figure 4, using the previously defined evolutionary receptor-like kinase sub-family groupings [6], there is no particular overall pattern of juxta-TM cysteine occurrence either between or within receptor-like kinase sub-families across different species. However, some orthologues, such as those for ERECTA, TMK3 or THESEUS, do appear to show conservation of juxta-TM cysteines across species. Interestingly the closest relatives of ERECTA, ERECTA-like1 and 2 (ERL1/2), do not have a juxta-TM cysteine in any species examined. This suggests that the juxta-TM cysteine of ERECTA appeared at the point when ERECTA diverged from the ERL1/2 clade, and has since been maintained in all extant angiosperm lineages. Given these data, and how S-acylation occurs sporadically throughout the Arabidopsis receptor-like kinase family tree (Supplemental figure 3), we propose that receptor-like kinase juxtaTM S-acylation has arisen and been lost multiple times over plant evolution and receptor-like kinase family diversification. Based on our data we propose that many juxta-TM cysteine-containing receptor-like kinases, such as AtFLS2, where orthologue juxta-TM cysteines are not conserved, may be fortuitously S-acylated but that S-acylation may become a fixed feature if it provides a novel beneficial function.

\section{Discussion}

Our previous work on S-acylation of AtFLS2 suggested that loss of S-acylation had a very mild effect on AtFLS2 function [5]. Since performing this work we have discovered that different epitope- tagged forms of AtFLS2 behave in an unpredictable manner, ranging from essentially fully functional to almost inactive [18]. The tag used in our original study [5], mGFP6, has a marked effect on AtFLS2 function when expressed at levels similar to endogenous AtFLS2 [18] but these effects can be masked by overexpression [5]. In light of this work, we reassessed our previous data using untagged forms of AtFLS2 lacking juxta-TM S-acylation, expressed at levels similar to endogenous AtFLS2. Using these lines we found that loss of AtFLS2 juxta-TM S-acylation had no discernible effect on any FLS2 mediated function or output tested. This surprising result led us to conclude that AtFLS2 juxta-TM S-acylation is likely fortuitous. This stands in stark contrast to the many documented examples of juxta-TM Sacylation being required for the trafficking, function or regulation of diverse single-pass transmembrane receptors in animals [2-4, 20].

S-acylation does not appear to be specified only by primary sequence; rather secondary and tertiary structure also likely govern whether S-acylation occurs. Common structural motifs for S-acylation 
include transmembrane domains followed by one or more cytosolic cysteine residues. Cysteines adjacent to a transmembrane domain likely evolved as an S-acylation motif as a result of the active site of S-acyl transferases being located at the membrane-cytosol interface, placing active site and Sacyl accepting cysteine in the same plane [23]. Juxta-TM cysteines are likely to arise comparatively often by chance as $13 / 61$ amino acid encoding codons ( $21 \%$ ) can alter to code for cysteine with only one base change. Furthermore, the amino acids encoded by these 13 codons (Phe, Tyr, Gly, Ser, Arg, Trp) are over-represented in $\alpha$-helical transmembrane domains and junctions between transmembrane domains and the aqueous environment [24]; the very region where S-acylated cysteines are found in receptor-like kinases. We propose that chance mutation may frequently result in cysteines occurring adjacent to transmembrane domains where they can be readily S-acylated by S-acyl transferases that recognise the "TM and juxta-TM cysteine" motif. Recognition of these new substrates by S-acyl transferases is eminently possible due to the common occurrence of this motif in pre-existing S-acylated proteins [20] and the rather lax specificity of many S-acyl transferases [25, 26]. Due to their reactivity, cysteine residues are not commonly found as surface exposed residues and tend to be under negative selection pressure unless they are required for a specific biological function [27]. However, S-acylation would essentially cap the cysteine residue and prevent reaction with other cellular constituents. As long as S-acylation does not negatively impact upon receptor-like kinase function the cysteine will therefore persist under neutral selection. We propose that S-acylation of AtFLS2 at Cys 830 and 831 falls into this category as they have no apparent effect on function and no AtFLS2 orthologue examined outside of the Brassicaceae possesses these sites, and absolute sequence and positional conservation within the Brassicacea is poor. This also seems to be the case for LYK4 as $\mathrm{S}$-acylation of LYK4 orthologues is not conserved. For instance, examination of the four dicot and three monocot species presented in supplemental figure 4 indicates that LYK4 orthologue S-acylation either arose in the last common ancestor of eudicots and monocots and was subsequently lost in $P$. trichocarpa, S. lycopersicon and Z. mays lineages or has arisen multiple times independently (Arabidopsis, C. melo, O. sativa and B. distachyon). If, however, S-acylation imparts a beneficial function or character to the receptor-like kinase, then it will be positively selected for and maintained over evolutionary time and distance. We hypothesise that this is the case for ERECTA. The ERECTAlike family of receptor-like kinases is present in all embryophytes, and gave rise to the ERECTA subclade [28] following the epsilon whole genome duplication event in the last common ancestor of all extant Angiosperms [29]. Interestingly, all ERECTA orthologues that we have examined (supplemental figure 3) contain positionally conserved juxta-TM cysteines suggesting that, from its point of origination, ERECTA was S-acylated. This is further supported by examination of ER and ERL orthologues from Amborella trichocarpa, considered to be the sister group to all other extant angiosperms [30], that follow the same pattern. We were unable to find evidence of ERECTA-like clade juxta-TM cysteines encoded in any gymnosperm genome. This would suggest that ERECTA S-acylation, unlike in AtFLS2, serves a purpose and we hypothesise that S-acylation enabled and characterised the emergence and neo-functionalisation of ERECTA from the ERECTA-like clade in a common ancestor of all extant sequenced angiosperms. We are currently investigating this intriguing role of S-acylation as a potential evolutionary driver in ERECTA function. This work has also revealed that all receptor-like kinases appear to be S-acylated at sites independent of any juxta-TM cysteines. This exciting finding indicates that S-acylation of receptor-like kinases is a general feature of the superfamily rather than a curious feature of a minority although the identity and role of these additional sites has yet to be determined. 


\section{Material and methods}

\section{Plant lines and growth conditions}

All Arabidopsis thaliana lines used in the work are Col-0 ecotype. fls2 (SAIL_691C4, [31]) and

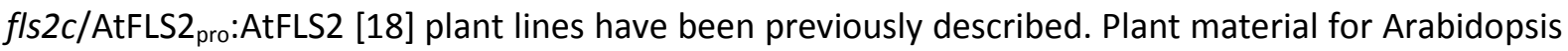
experiments was grown on $0.5 x$ Murashige \& Skoog (MS) medium containing $0.8 \%(\mathrm{w} / \mathrm{v})$ agar. Seeds were stratified for $48 \mathrm{~h}$ at $4^{\circ} \mathrm{C}$ and germinated under a $16 / 8 \mathrm{~h}$ light/dark cycle at $20^{\circ} \mathrm{C}$ in MLR-350 growth chamber (Panasonic). Nicotiana benthamiana plants expressing mOrange-LTI6b fluorescent protein as a plasma membrane marker (mOrg-LTI; [32]) were used for confocal imaging. Transgenic and wild-type Nicotiana benthamiana plants were grown in commercial compost and maintained under glasshouse conditions of $16 / 8 \mathrm{~h}$ light/ dark cycle, daytime temperature $26^{\circ} \mathrm{C}$ maximum, nighttime $22^{\circ} \mathrm{C}$ for $4-5$ weeks before use.

\section{Constructs}

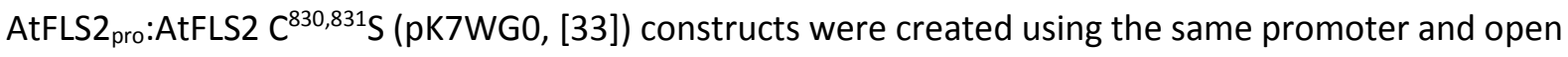
reading frame as found in fls2/AtFLS2 pro:AtFLS2 described previously [18]. Cysteine codon mutants and reading frame truncations were generated using Q5 site-directed mutagenesis (NEB). Transgenic Arabidopsis plant lines were generated by Agrobacterium tumefaciens-mediated floral dip transformation [34] and selected for homozygosity at T3. Additional receptor-like kinases were cloned from genomic Col-O DNA without a stop codon into pENTR D-TOPO before sequencing and recombination into pK7FWG2 [33] or pGWB17 [35] for expression from the 355 promoter as Cterminal EGFP or 4xMYC fusions respectively.

\section{Transient expression in $\mathbf{N}$. benthamiana}

Leaves of 4-5 week old wild type and mOrg-LTI [32] expressing Nicotiana benthamiana plants were syringe infiltrated with Agrobacterium tumefaciens GV3101 pMP90 containing expression plasmids for each receptor-like kinase alongside $\mathrm{p} 19$, each at an $\mathrm{OD}_{600}$ of 0.1 . Plants were maintained under standard growth conditions for 48-60h before use in experiments.

\section{Gene expression analysis}

Gene expression level analysis was carried out as previously described [18, 36]. Gene expression was analysed by quantitative reverse transcription PCR. 10 seedlings of each genotype 10 days postgermination were treated with $1 \mu \mathrm{M}$ flg22 for the indicated times in $2 \mathrm{~mL} 0.5 \mathrm{x}$ MS liquid medium. RNA was extracted using an RNeasy mini kit with on-column DNAse digestion according to manufacturer's instructions (QIAgen). $2 \mu \mathrm{g}$ RNA was reverse transcribed using a high capacity CDNA reverse transcription kit (Applied Biosystems). Expression levels were normalised against PEX4 (At5G25760, [37]). Relative quantification was calculated using the $\Delta \Delta C_{T}$ method [38]. Error bars represent $R Q_{\text {MAx }}$ and $R Q_{\text {MIN }}$ from three technical replicates and constitute a $95 \%$ confidence interval, according to a Student's t-test.

\section{MAPK activation}

MAPK activation assays were performed as described previously [39]. Six seedlings of each genotype 10 days post-germination were transferred to 12 -well plates containing $2 \mathrm{~mL} 0.5 x \mathrm{MS}$ liquid media for $24 \mathrm{~h}$ and then treated with $100 \mathrm{nM}$ flg22 for the indicated times in $2 \mathrm{~mL} 0.5 \mathrm{x}$ MS medium. 30 $\mu \mathrm{g}$ total protein was loaded per sample. 


\section{Immunoblot analysis of protein levels}

Proteins were extracted from pools of seedlings as previously described [22] separated by SDS-PAGE and transferred to PVDF membrane using wet transfer methods and blotted for active and total MAPK [39] or AtFLS2 [22]. Antibodies used were as follows: $\alpha$-p42/44 MAPK: CST (\#9102); $\alpha$-MPK6: SigmaAldrich (A7104); $\alpha$-EGFP: Roche (11867423001); $\alpha$-MYC: Thermo (MA1-21316).

\section{Callose staining}

Callose staining was carried out as previously described [40]. Seedlings were destained overnight before aniline blue stain was applied for $3 \mathrm{~h}$ in the dark. Callose images were obtained from cotyledons 10 days post-germination treated for $12 \mathrm{~h}$ with $1 \mu \mathrm{m}$ flg22 or water. Cotyledons were mounted in $50 \%$ glycerol and imaged using a LEICA DMLFS microscope with a $5 x$ objective lens and UV D filter. Callose was quantified using an image processing macro in FIJI [41] adapted from [42]. Colour images were converted to 8-bit greyscale before analysis.

\section{Seedling growth inhibition}

Seedlings growth inhibitions were carried out as previously described $[18,43]$. Four days postgermination, 10 seedlings of each genotype were transferred to 12 -well plates containing $2 \mathrm{~mL} 0.5 \mathrm{x}$ MS liquid media with or without $1 \mu \mathrm{M}$ flg22, ensuring cotyledons were not submerged. Seedlings were grown for a further 10 days, blotted dry and the fresh weight of pooled seedlings in each genotype for each treatment weighed and an average calculated.

\section{S-acylation assay}

$S$-acylation assays were carried out as previously described [22]. Briefly samples were ground to a fine powder in liquid nitrogen and resuspended in $2 \mathrm{~mL}$ lysis buffer $(50 \mathrm{mM}$ Tris $\mathrm{HCl} \mathrm{pH} \mathrm{7.2,} 5 \mathrm{mM}$ EDTA, $150 \mathrm{mM} \mathrm{NaCl}, 2.5 \%$ SDS, $10 \mathrm{mM} \mathrm{N}$-ethylmaleimide, $5 \mu \mathrm{l} . \mathrm{ml}^{-1}$ protease inhibitor [P9599, Sigma-Aldrich], $100 \mathrm{mg}$ PVPP). Samples were mixed gently for 10 minutes at room temperature and centrifuged at $16,000 \mathrm{xg}$ for 1 minute. The supernatant was retained and total protein concentration was determined by BCA assay (ThermoFisher). $1 \mathrm{mg}$ of protein was diluted to a final volume of $1 \mathrm{~mL}$ with lysis buffer (no PVPP) and incubated for $1 \mathrm{~h}$ at room temperature with gentle mixing. $12 \mu \mathrm{l} 2,3$-dimethyl 1,3butadiene was added to each sample and incubated at $25^{\circ} \mathrm{C}$ in a shaking incubator for $1 \mathrm{~h}$ to remove unreacted NEM. $1 / 10^{\text {th }}$ volume chloroform was added to each sample, briefly vortexed and centrifuged to achieve phase partitioning. The upper phase was retained split in two $0.5 \mathrm{~mL}$ samples; one treated with $1 \mathrm{M}$ hydroxylamine $\mathrm{pH} 7.2$ and the other with an equal volume of water. $10 \mu$ loading control was then taken from each sample and incubated at RT for 1 hour before being combined with $10 \mu \mathrm{l} 2 \mathrm{x}$ reducing SDS-PAGE loading buffer. $20 \mu \mathrm{l}$ equivalent of a $50 \%$ slurry of thiopropyl Sepharose $6 \mathrm{~b}$ beads pre-swollen in lysis buffer (no NEM) was added to remaining samples and samples incubated for $1 \mathrm{~h}$ with gentle mixing for $1 \mathrm{~h}$ at room temperature. Beads were washed three times in lysis buffer (no NEM) for five minutes each and the supernatant aspirated after the final wash. Proteins were eluted in $25 \mu \mathrm{l} 2 \mathrm{x}$ reducing SDS-PAGE loading buffer at $37^{\circ} \mathrm{C}$ for 30 minutes with agitation.

\section{Confocal imaging}

Cells were imaged using a Nikon A1R confocal laser scanning microscope mounted on an NiE upright microscope fitted with an NIR Apo 40x 0.8W water dipping lens and GaASP detectors. Images are false colour single confocal sections or maximum projections of Z-stacks produced using NIS-elements AR software. GFP was excited at $488 \mathrm{~nm}$ with emissions gathered at 500-530 nm with simultaneous 
collection of chlorophyll fluorescence at $663-738 \mathrm{~nm}$. mOrange was excited sequentially at $561 \mathrm{~nm}$ with emissions gathered at 570-620 nm.

\section{Bioinfomatic analyses}

Biomart [44] was used to query EnsemblPlants [45] and extract the sequences of the 609 Arabidopsis RLK superfamily proteins defined in the work of Shiu and Bleecker [6]. Custom Perl scripts were used to extract sequences of the TM domain as defined in the ARAMEMNON database [21] plus 10 residues after the end of the TM domain. Custom scripts were then used to identify those proteins with cysteines within the region $-1,+5$ of the end of the TM domain. Figures were generated using custom scripts and ESPript3 [46].

\section{Accession numbers}

Accession numbers are as follows: FLS2, At5g46330; ER, At2g26330; LYK4, At2g23770; FER, At3g51550; BRI1, At4g39400; FEI1, At1g31420; ERL1, At5g62230; NHL10, At2g35980; WRKY40, ; PHI1, At1g35140; PR1, At2g14610; PEX4, At5g25760; MPK6, At2g43790; and MPK3, At3g45640.

\section{Acknowledgements}

We would like to thank Dr. Nana Keinath and Profs. Antje Heese, Silke Robatzek and Cyril Zipfel for helpful discussions during the preparation of this manuscript. This work was funded by UK Biotechnology and Biological Sciences Research Council grants BB/M024911/1 and BB/M010996/1 to P.A.H.

\section{Author contributions}

PAH conceived and developed the work. $\mathrm{CHH}, \mathrm{DT}, \mathrm{KMW}, \mathrm{SJ}, \mathrm{KL}$ and PAH performed the experiments and analysed the results. PAH and $\mathrm{CHH}$ wrote the manuscript with input from DT, KMW, SJ and KL.

\section{Competing interest}

The authors declare no competing interests.

\section{References}

1. Ren, J., et al., CSS-Palm 2.0: an updated software for palmitoylation sites prediction. Protein Eng Des Sel, 2008. 21(11): p. 639-44.

2. Chesarino, N.M., et al., Chemoproteomics reveals Toll-like receptor fatty acylation. BMC Biol, 2014. 12: p. 91.

3. Abrami, L., S.H. Leppla, and F.G. van der Goot, Receptor palmitoylation and ubiquitination regulate anthrax toxin endocytosis. J Cell Biol, 2006. 172(2): p. 309-20.

4. Abrami, L., et al., Palmitoylation and ubiquitination regulate exit of the Wnt signaling protein LRP6 from the endoplasmic reticulum. Proc Natl Acad Sci U S A, 2008. 105(14): p. 5384-9.

5. Hemsley, P.A., et al., A proteomic approach identifies many novel palmitoylated proteins in Arabidopsis. New Phytologist, 2013. 197(3): p. 805-814.

6. Shiu, S.H. and A.B. Bleecker, Receptor-like kinases from Arabidopsis form a monophyletic gene family related to animal receptor kinases. Proc Natl Acad Sci U S A, 2001. 98(19): p. 10763-8.

7. Friedrichsen, D.M., et al., Brassinosteroid-insensitive-1 is a ubiquitously expressed leucine-rich repeat receptor serine/threonine kinase. Plant Physiol, 2000. 123(4): p. 1247-56.

8. Torii, K.U., et al., The Arabidopsis ERECTA gene encodes a putative receptor protein kinase with extracellular leucine-rich repeats. Plant Cell, 1996. 8(4): p. 735-46. 
9. Huck, N., et al., The Arabidopsis mutant feronia disrupts the female gametophytic control of pollen tube reception. Development, 2003. 130(10): p. 2149-59.

10. Schoenaers, S., et al., The Auxin-Regulated CrRLK1L Kinase ERULUS Controls Cell Wall Composition during Root Hair Tip Growth. Curr Biol, 2018. 28(5): p. 722-732 e6.

11. He, Z.H., M. Fujiki, and B.D. Kohorn, A cell wall-associated, receptor-like protein kinase. J Biol Chem, 1996. 271(33): p. 19789-93.

12. Song, W.Y., et al., A receptor kinase-like protein encoded by the rice disease resistance gene, Xa21. Science, 1995. 270(5243): p. 1804-6.

13. Gomez-Gomez, L. and T. Boller, FLS2: an LRR receptor-like kinase involved in the perception of the bacterial elicitor flagellin in Arabidopsis. Mol Cell, 2000. 5(6): p. 1003-11.

14. Zipfel, C., et al., Perception of the bacterial PAMP EF-Tu by the receptor EFR restricts Agrobacterium-mediated transformation. Cell, 2006. 125(4): p. 749-60.

15. Wan, J., et al., LYK4, a lysin motif receptor-like kinase, is important for chitin signaling and plant innate immunity in Arabidopsis. Plant Physiol, 2012. 160(1): p. 396-406.

16. Boyle, P.C., et al., Detecting N-myristoylation and S-acylation of host and pathogen proteins in plants using click chemistry. Plant Methods, 2016. 12: p. 38.

17. Curtis, M.D. and U. Grossniklaus, A gateway cloning vector set for high-throughput functional analysis of genes in planta. Plant Physiol, 2003. 133(2): p. 462-9.

18. Hurst, C.H., et al., Variable Effects of C-Terminal Fusions on FLS2 Function: Not All Epitope Tags Are Created Equal. Plant Physiol, 2018. 177(2): p. 522-531.

19. Nühse, T.S., et al., Microbial elicitors induce activation and dual phosphorylation of the Arabidopsis thaliana MAPK 6. J Biol Chem, 2000. 275(11): p. 7521-6.

20. Blaskovic, S., M. Blanc, and F.G. van der Goot, What does S-palmitoylation do to membrane proteins? FEBS J, 2013. 280(12): p. 2766-74.

21. Schwacke, R., et al., ARAMEMNON, a novel database for Arabidopsis integral membrane proteins. Plant Physiol, 2003. 131(1): p. 16-26.

22. Hurst, C.H., et al., Maleimide scavenging enhances determination of protein S-palmitoylation state in acyl-exchange methods. Biotechniques, 2017. 62(2): p. 69-75.

23. Rana, M.S., et al., Fatty acyl recognition and transfer by an integral membrane Sacyltransferase. Science, 2018. 359(6372).

24. Ulmschneider, M.B. and M.S. Sansom, Amino acid distributions in integral membrane protein structures. Biochim Biophys Acta, 2001. 1512(1): p. 1-14.

25. Jennings, B.C. and M.E. Linder, DHHC protein S-acyltransferases use similar ping-pong kinetic mechanisms but display different acyl-CoA specificities. J Biol Chem, 2012. 287(10): p. 723645.

26. Roth, A.F., et al., Global analysis of protein palmitoylation in yeast. Cell, 2006. 125(5): p. 100313.

27. Marino, S.M. and V.N. Gladyshev, Cysteine function governs its conservation and degeneration and restricts its utilization on protein surfaces. J Mol Biol, 2010. 404(5): p. 902-16.

28. Zhang, Y., et al., Phylogenetic and CRISPR/Cas9 Studies in Deciphering the Evolutionary Trajectory and Phenotypic Impacts of Rice ERECTA Genes. Front Plant Sci, 2018. 9: p. 473.

29. Jiao, Y., et al., Ancestral polyploidy in seed plants and angiosperms. Nature, 2011. 473(7345): p. $97-100$.

30. Amborella Genome, P., The Amborella genome and the evolution of flowering plants. Science, 2013. 342(6165): p. 1241089.

31. Zipfel, C., et al., Bacterial disease resistance in Arabidopsis through flagellin perception. Nature, 2004. 428(6984): p. 764-7.

32. Wright, K.M., et al., Differences in internalization and growth of Escherichia coli 0157:H7 within the apoplast of edible plants, spinach and lettuce, compared with the model species Nicotiana benthamiana. Microb Biotechnol, 2017. 10(3): p. 555-569. 
33. Karimi, M., D. Inze, and A. Depicker, GATEWAY vectors for Agrobacterium-mediated plant transformation. Trends Plant Sci, 2002. 7(5): p. 193-5.

34. Clough, S.J. and A.F. Bent, Floral dip: a simplified method for Agrobacterium-mediated transformation of Arabidopsis thaliana. Plant J, 1998. 16(6): p. 735-43.

35. Nakagawa, T., et al., Development of series of gateway binary vectors, $p G W B s$, for realizing efficient construction of fusion genes for plant transformation. J Biosci Bioeng, 2007. 104(1): p. 34-41.

36. Hemsley, P.A., et al., The MED16, MED14 and MED2 subunits of the Arabidopsis Mediator complex control recruitment of Mediator and RNA polymerase II to CBF-responsive coldregulated genes. The Plant Cell, 2014.

37. Wathugala, D.L., et al., The Mediator subunit SFR6/MED16 controls defence gene expression mediated by salicylic acid and jasmonate responsive pathways. New Phytologist, 2012. 195(1): p. 217-230.

38. Schmittgen, T.D. and K.J. Livak, Analyzing real-time PCR data by the comparative $C(T)$ method. Nat Protoc, 2008. 3(6): p. 1101-8.

39. Schwessinger, B., et al., Phosphorylation-dependent differential regulation of plant growth, cell death, and innate immunity by the regulatory receptor-like kinase BAK1. PLoS Genet, 2011. 7(4): p. e1002046.

40. Schenk, S.T. and A. Schikora, Staining of Callose Depositions in Root and Leaf Tissues. Bioprotocol, 2015. 5(6): p. e1429.

41. Schindelin, J., et al., Fiji: an open-source platform for biological-image analysis. Nat Methods, 2012. 9(7): p. 676-82.

42. Zavaliev, R. and B.L. Epel, Imaging callose at plasmodesmata using aniline blue: quantitative confocal microscopy. Methods Mol Biol, 2015. 1217: p. 105-19.

43. Gomez-Gomez, L., G. Felix, and T. Boller, A single locus determines sensitivity to bacterial flagellin in Arabidopsis thaliana. Plant J, 1999. 18(3): p. 277-84.

44. Smedley, D., et al., BioMart--biological queries made easy. BMC Genomics, 2009. 10: p. 22.

45. Kersey, P.J., et al., Ensembl Genomes 2018: an integrated omics infrastructure for nonvertebrate species. Nucleic Acids Res, 2018. 46(D1): p. D802-D808.

46. Robert, $X$. and P. Gouet, Deciphering key features in protein structures with the new ENDscript server. Nucleic Acids Res, 2014. 42(Web Server issue): p. W320-4. 